\title{
Composición nutricional del sedimento en estanques con tilapia roja
}

\author{
Nutritional composition of the sediment in ponds \\ with red tilapia
}

\section{Composição nutricional do sedimento nos viveiros de tilápia vermelha}

\author{
Martha Yossa $^{1 *}$, Gilma Hernández-Arevalo ${ }^{2 *}$, Walter Vásquez-Torres ${ }^{3 *}$ \\ Zootecnista, MSc, PhD \\ 2 MVZ, Especialista, MSc \\ 3 Biólogo, MSc, PhD \\ * Instituto de Acuicultura de los Llanos, grupo de investigación en alimentación y nutrición de organismos acuáticos- \\ GRANAC- Línea: Dinámica de nutrientes \\ Email: granac.iall@gmail.com
}

Recibido: septiembre 4 de 2012 Aceptado: noviembre 29 de 2012

\begin{abstract}
Resumen
Materia orgánica $(\mathrm{MO}), \mathrm{pH}$, fosforo disponible, macro y micronutrientes fueron analizados en el sedimento superficial de estanques comerciales de tilapia roja durante dos ciclos productivos. En ocho de los catorce nutrientes no hubo diferencia significativa ( $\mathrm{P}>0.05$ ) entre ciclos, sin embargo, ocurrió incremento en $\mathrm{MO}, \mathrm{Ca}, \mathrm{Mg}, \mathrm{K}$ y Mn, así como disminución en $\mathrm{Al}$, Cu y B durante el segundo ciclo. Aunque el $\mathrm{pH}$ aumentó, no hubo diferencia significativa $(\mathrm{P}>0.05)$, en cuanto que las concentraciones de $\mathrm{P}$ disponible, $\mathrm{Na}$, Fe y $\mathrm{Zn}$ aumentaron, con diferencia altamente significativa $(\mathrm{P}<0.01)$. Fueron determinadas correlaciones positivas altamente significativas $(\mathrm{P}<0.01)$ entre Fe y $\mathrm{Zn}$; así como entre materia orgánica y $\mathrm{P}$ con $\mathrm{Ca}$, Fe y $\mathrm{Zn}$. A pesar del constante suministro de aditivos en esta granja para mantener un sistema de aguas verdes, la MO no fue superior a 3\% lo que reflejó un adecuado proceso de mineralización; igualmente los valores de macro y micro nutrientes en términos generales estuvieron estables. Este equilibrio en el sistema pudo ser favorecido por el uso de aireadores, la eficiencia de los probióticos y la acción bioturbadora de la tilapia.
\end{abstract}

Palabras clave: materia orgánica, $\mathrm{pH}$, macronutrientes, micronutrientes, Orinoquia,

\begin{abstract}
Organic matter $(\mathrm{OM}), \mathrm{pH}$, available phosphorus, macro and micronutrients were analyzed in surface sediments of commercial ponds of red tilapia for two cycles. In eight of the fourteen nutrients no significant difference $(\mathrm{P}>0.05)$ between cycles, however, did increase in the $\mathrm{OM}, \mathrm{Ca}, \mathrm{Mg}, \mathrm{K}$ and $\mathrm{Mn}$, but decrease in $\mathrm{Al}, \mathrm{Cu}$ and $\mathrm{B}$ in the second cycle. Although the $\mathrm{pH}$ increased, there was no significant difference ( $P>0.05)$, in that the concentrations of available $P, N a, F e$ and $Z n$ increased, with highly significant difference $(P<0.01)$. There was also highly significant positive correlations $(P<0.01)$ of Fe wiht $Z n$, and $\mathrm{OM}$ and $\mathrm{P}$ with $\mathrm{Ca}$, Fe and $\mathrm{Zn}$. Despite the constant supply of additives in this farm to maintain a system of green water, the OM was greater than $3 \%$ which reflected a proper process of mineralization, also the values of macro and micro nutrients
\end{abstract}


were generally stable. This balance in the system could be enhanced by the uses of aerators, efficient biological activity of probitic and bioturbation action of tilapia.

Key words: organic matter, $\mathrm{pH}$, macronutrients, micronutrients, orinoquia

\begin{abstract}
Resumo
Matéria orgânica $(\mathrm{MO}), \mathrm{pH}$, fósforo disponível, macro e micronutrientes foram analisados no sedimento da superfície de viveiros comerciais de tilápia vermelha durante dois ciclos produtivos. Em oito de quatorze nutrientes no tiveram diferença significativa $(\mathrm{P}>0.05)$ nos ciclos, contudo, ocorreu incremento no $\mathrm{MO}, \mathrm{Ca}, \mathrm{Mg}, \mathrm{K}$ e $\mathrm{Mn}$, assim como diminuição no $\mathrm{Al}$, Cu e $\mathrm{B}$ durante o segundo ciclo. $\mathrm{O}$ pH aumentou mas sem diferença significativa ( $\mathrm{P}>0.05)$, entre tanto, as concentrações de $\mathrm{P}$ disponível, $\mathrm{pH}, \mathrm{Na}$, Fe e $\mathrm{Zn}$ aumentaram com diferenças altamente significativa $(\mathrm{P}<0.01)$. Assim mesmo, houve correlações positivas altamente significativas $(\mathrm{P}<0.01)$ do Fe com Zn; e matéria orgânica e P com Ca, Fe e Zn. Apesar do constante subministro nesta granja de aditivos para manter o sistema de águas verdes, a MO não foi superior ao 3\% o que reflete um adequado processo de mineralização; além disso, os valores de macro e micro nutrientes em términos gerais estiveram estáveis. O equilíbrio no sistema pode ter sido favorecido pelo uso de aeradores, a atividade biologicamente eficiente dos probióticos e da ação bioturbadora da tilápia.
\end{abstract}

Palavra chave: matéria orgânica, $\mathrm{pH}$, macronutrientes, micronutrientes, orinoquia

\section{Introducción}

La piscicultura en Colombia se basa principalmente en el cultivo de tilapia en jaulas y en estanques, representando alrededor del $60 \%$ de la producción nacional y teniendo al departamento del Meta como tercer productor de tilapia roja (Oreochromis spp.) (Espinal et al., 2005; MADR, 2010), cultivada en estanques excavados en tierra. Estos sistemas de producción son los más comunes en la acuicultura de agua dulce Steeby et al. (2004), aunque en ellos, el suelo circundante afecte significativamente el abastecimiento de nutrientes solubles a través del sedimento, debido al ingreso de sólidos derivados de la erosión (macro y micronutrientes) y de la materia orgánica (dieta comercial, fitoplancton y sólidos excretados por los peces, principalmente), liberan nutrientes y constituyen un medio para el desarrollo de organismos bentónicos y bacterias (Boyd, 1995).

El sedimento refleja la composición de los materiales alóctonos y autóctonos, la velocidad con que se acumulan y la actividad biológica; contiene entre 20 y $80 \%$ de agua que ocupa las cavidades entre los materiales sólidos, creando así una zona de intercambio de nutrientes en la interfase agua-sedimento; los compuestos a base de nitrógeno, fósforo y carbono adicionados a los estanques, aumentan la producción y concentración de materia orgánica (Boyd et al., 2002; Jiménez-Tenorio et al., 2007). Así, al aumentar la capa de sedimento a expensas de la materia orgánica, se crea un ambiente de acción microbiana (Lehmann, 2005), donde se degradan materiales de alto peso molecular, como el selenio cuya degradación se incrementa dependiendo de la profundidad del sedimento, en $50 \%$ como materia orgánica mineralizada y $35 \%$ como seleniuro orgánico (Meseck y Cutter, 2012); también se aumentan la tasa de respiración que implica consumo de oxígeno, se crean zonas anaeróbicas causantes de estrés a los organismos cultivados y se afecta la calidad del agua; este proceso describe la dinámica de los nutrientes en los sistemas acuáticos (Förstner, 1990).

En la capa superficial de sedimento, la concentración de nutrientes es 1000:1 con relación a la columna de agua (Avnimelech y Ritvo, 2003) y se producen ácidos orgánicos que disminuyen el $\mathrm{pH}$ del agua de poro, aumenta el contenido de sulfuros, amonio, iones de hierro y otros iones o moléculas (Vita et al., 2002; Boyd, 2008; Parra y Espinosa, 2008;). En esta dinámica, los nutrientes minerales esenciales para el fitoplancton son suplidos por el suelo (Boyd, 1995) y su demanda es mayor para mantener un sistema de aguas verdes, que a su vez reduce los niveles de nitrógeno y promueve la producción de oxigeno (Poleo et al., 2011).

Hay cinco mecanismos para la acumulación de nutrientes en el sedimento y posterior disponibilidad en la columna de agua: 1 . Adsorción de metales, principalmente cationes intercambiables como $\mathrm{Na}, \mathrm{K}, \mathrm{Ca}$ y $\mathrm{Mg}$, que al estar disueltos en el agua quedan suspendidos sobre las finas partículas de arcilla $(<2 \mu \mathrm{m})$ de carga eléctrica negativa (Boyd, 2008); 2. Precipitación de compuestos metálicos, sedimentación de los minerales bajo la forma de hidróxidos, como el de aluminio $\mathrm{Al}(\mathrm{OH})$ que puede disolverse tanto en condiciones ácidas como básicas y dejan libres los iones más solubles; 3. Co- precipitación de metales con sales inorgánicas originadas en el suelo, mediante entrampamiento por hidróxidos de hierro, óxidos de manganeso y carbonatos; 4. Asociación con moléculas orgánicas e incor- 
poración en minerales cristalinos y 5. Potencial redox del fondo y actividad microbiana, que determinan las formas de asociación de los metales con la matriz de sedimento (Krebs, 2003), además, permanentemente el sedimento actúa como una reserva tampón de nutrientes para la columna de agua, porque por un lado, amortigua los aumentos de nutrientes en el medio, provenientes de los aportes directos y/o de la descomposición de materia orgánica, reteniendo una parte de los mismos y por otro lado, porque compensan los déficits de nutrientes en periodos de alta demanda biológica, liberando parte de estas formas retenidas (Boyd, 2008).

Existen otras fracciones no biodisponibles entre las que se encuentran metales precipitados, enlazados a sulfuros, a materia orgánica meteorizada y a minerales secundarios, los cuales están fuertemente enlazados al sedimento y no pueden ser fácilmente re suspendidos en el agua (Parra y Espinosa, 2008) sin la actividad biológica y la acción bioturbadora de especies como la tilapia (Adámek y Maršálek, 2012).

Las prácticas inadecuadas de manejo como suministro excesivo de abono, fertilizantes y alimento, entre otros, alteran la dinámica e incrementan la carga de nutrientes y la concentración de amonio que ocasiona bajas de oxígeno y empobrece las condiciones para los sedimentos del fondo del estanque. Este cuadro afecta inicialmente a los macroinvertebrados bentónicos que juegan un papel importante en el reciclaje de nutrientes, fundamental para la estabilidad del sistema (Adedeji et al., 2012; Gu et al., 2012). Sin embargo, por el enriquecimiento de nutrientes, es considerado el uso alternativo de los sedimentos acuícolas como fertilizantes y/o acondicionadores de suelos agrícolas para incrementar la concentración de nutrientes (Ihejirika et al., 2012; Leue y Lang, 2012), y aunque con esto se solucionaría un problema de la acuicultura, como es la acumulación de sedimentos (FAO, 2006), no necesariamente mejoraría la calidad del suelo ya que según Boyd (2002) el suelo del fondo de los estanques no difiere significativamente del suelo de la región.

Los suelos del departamento del Meta son de origen sedimentario, de diferente composición mineralógica y edad de las rocas, que en forma natural y con participación de factores externos, como el clima, causa diferentes grados de erosión y sedimentación (Botero, 1999). Por ejemplo, Castilla La Nueva, en el departamento del Meta donde se realizó el presente estudio, está ubicada en una terraza baja, originada por acción fluvial del río Guamal, el cual aporta sedimentos provenientes de la Cordillera Oriental, de mineralogía constituida principalmente por arcillas alumino-silicatadas y en la fracción arena, conformada por cuarzos, cuyas características químicas son de suelos muy ácidos; de bajas concentraciones de bases, fósforo y elementos menores, sin embargo sobresalen Fe y Al aportados por minerales que al meteorizarse los liberan (Botero, 1999; Zapata, 2006). El objetivo de este estudio fue determinar el valor nutricional del sedimento en términos de materia orgánica, fosforo disponible, macro y micronutrientes, como paso inicial para entender la dinámica de los nutrientes en el sistema productivo piscícola regional.

\section{Materiales y metodos}

En una granja ubicada en Castilla La Nueva $\left(74^{\circ} 04^{\prime} 58^{\prime \prime}\right.$ W; $\left.04^{\circ} 35^{\prime} 56^{\prime \prime} \mathrm{N}\right)$, con temperatura promedio $26^{\circ} \mathrm{C}$ y $350 \mathrm{msnm}$, fue analizado el sedimento del fondo de los estanques durante dos ciclos productivos, para este fin se seleccionaron al azar, tres estanques excavados en tierra, dos de una hectárea y otro de $6600 \mathrm{~m}^{2}$, cultivados con tilapia (Oreochromis spp). Los estanques, durante los dos ciclos estudiados, fueron manejados bajo las prácticas ya establecidas por el productor, que incluyeron: secado de 3 a 8 días, encalado con 40 g/ $\mathrm{m}^{2}$ de cal viva y $80 \mathrm{~g} / \mathrm{m}^{2}$ de cal dolomita, adición de yeso, cloruro de potasio, urea, melaza y probióticos en cantidades predeterminadas para mantener un sistema de "aguas verdes"; uso de tres aireadores de paleta de $2 \mathrm{Hp}$ por estanque, accionados ante la alarma de baja de oxígeno (3 ppm), siembra de alevinos (2.5 g士 0.02), a una densidad final de 5 peces $/ \mathrm{m}^{2}$ y suministro de alimento comercial acorde con la tabla de alimentación establecida para la granja.

En el primer ciclo (2008) todos los alevinos fueron sembrados y mantenidos en un único estanque hasta alcanzar un peso de 50 a $60 \mathrm{~g}$ y ser trasladados a los estanques donde cumplirían el ciclo de cultivo. En el segundo ciclo (2010), los alevinos fueron sembrados en hapas, por los primeros 20 días, continuando su ciclo de cultivo en el mismo estanque.

Durante cada ciclo de cultivo se realizaron cinco muestreos, con un intervalo entre muestreos de un mes aproximadamente. En cada muestreo el sedimento fue recolectado de la capa superficial del fondo del estanque, en tres puntos (entrada, centro y salida), usando colectores construidos en tubo PVC de 2.5 $\mathrm{cm}$ de diámetro y $15 \mathrm{~cm}$ de largo (con orificios alineados para eliminar el exceso de agua), acoplados a un tubo de $1.5 \mathrm{~m}$ de longitud, para llegar hasta el fondo del estanque desde un bote sin disturbar el medio. Las muestras homogenizadas, empacadas en bolsas plásticas e debidamente identificadas fueron transportadas refrigeradas al laboratorio de Ecología Acuática de la Universidad de los Llanos, donde se secaron en estufa 
a $55^{\circ} \mathrm{C}$ por 72 horas, posteriormente fueron maceradas, empacadas (100 g) en bolsas de papel, y enviadas al Laboratorio de Suelos de la Universidad de los Llanos, para análisis completo que incluyó: determinación de materia orgánica $(\mathrm{MO})$ por el método de Walkley Black, potencial de hidrógeno proporción 1:1 suelo-agua; azufre $(\mathrm{S})$ por extracción con fosfato monobásico de calcio $0.008 \mathrm{M}$; bases intercambiables calcio (Ca), magnesio $(\mathrm{Mg})$, sodio $(\mathrm{Na})$ y potasio $(\mathrm{K})$, con acetato de amonio $1 \mathrm{~N}$ neutro a $\mathrm{pH}$ 7.0; elementos menores, cobre $(\mathrm{Cu})$, hierro $(\mathrm{Fe})$, manganeso $(\mathrm{Mn})$, y zinc $(\mathrm{Zn})$ por el método ácido dietilen-triamino-penta-acético (DTPA); aluminio (Al) con $\mathrm{KCl} 1 \mathrm{~N}$; boro (B) en frio con $\mathrm{HCl} 0.05 \mathrm{M}$ y fósforo disponible ( $\left.\mathrm{P}-\mathrm{d}_{-}\right)$con Solución Bray II (APHA, 2005).

Para el análisis de las variables $\mathrm{MO}, \mathrm{P}-\mathrm{d}, \mathrm{pH}, \mathrm{Ca}, \mathrm{Mn}$, $\mathrm{Al}, \mathrm{Cu}, \mathrm{Fe}, \mathrm{Zn}$ y B fue realizado un análisis de varianza, incluyendo dentro del modelo el efecto fijo del ciclo de cultivo y el efecto aleatorio del día de muestreo anidado dentro del ciclo de cultivo, en el caso de $\mathrm{Al}, \mathrm{Fe}$ y Zn fue necesaria la transformación $\log _{10}$ para garantizar la homogeneidad de varianzas (Test de Levene, $\alpha=0.05)$ y la normalidad del error (Test de Shapiro-Wi$I k, \alpha=0.05)$ (Quin y Keough, 2002). Para las variables $\mathrm{Mg}, \mathrm{K}$ y $\mathrm{Na}$ el efecto del ciclo de cultivo fue evaluado mediante la prueba de Kruskal-Wallis debido a la heterocedasticidad y no normalidad del error que no fueron superados mediante la transformación $\log _{10}$. Para identificar correlación entre las variables, se utilizó el coeficiente $t$ de Kendall. Los análisis estadísticos fueron realizados mediante al paquete estadístico $R$.

\section{Resultados}

El resultado de cada variable analizada en los tres estanques, es presentado como el valor medio de los cinco muestreos por ciclo de producción (Tabla 1). En ocho de los 14 nutrientes analizados no hubo diferencia significativa $(P>0.05)$ entre los dos ciclos productivos, sin embargo se observó incremento en las concentraciones de $\mathrm{MO}, \mathrm{Ca}, \mathrm{Mg}, \mathrm{K}$ y $\mathrm{Mn}$, así como disminución en $\mathrm{Al}$, Cu y $\mathrm{B}$ durante el segundo ciclo. El $\mathrm{pH}$ y las concentraciones de $\mathrm{P}_{-\mathrm{d}}, \mathrm{Na}$, Fe y $\mathrm{Zn}$ también se incrementaron en el segundo ciclo, con diferencia significativa en el caso del $\mathrm{pH}(\mathrm{p}<0.05)$ y altamente significativa $(\mathrm{P}<0.01)$ para las concentraciones mencionadas (Tabla 1). De otro lado, se evidenciaron correlaciones positivas altamente significativas $(\mathrm{P}<0.01)$ entre Fe y Zn, así como en $\mathrm{MO}$ y P-d con $\mathrm{Ca}$, Fe y Zn; además fueron determinadas correlaciones significativas $(\mathrm{P}<0.05)$ tanto positivas como negativas entre los diferentes elementos analizados (Tabla 2).

\section{Discusión}

La acumulación de materia orgánica es apuntada como uno de los mayores efectos de la acuicultura

Tabla 1. Composición nutricional (media \pm SD) del sedimento piscícola en tres estanques de cultivo de tilapia roja, durante dos ciclos productivos

\begin{tabular}{|c|c|c|c|c|c|c|c|}
\hline \multirow{3}{*}{$\begin{array}{c}\text { Variable } \\
\mathrm{MO}(\%)\end{array}$} & \multicolumn{6}{|c|}{ Ciclo de cultivo (5 meses) } & \multirow{3}{*}{$\frac{\text { Significancia }}{\text { Ns }}$} \\
\hline & \multicolumn{3}{|c|}{$1(n=5)$} & \multicolumn{3}{|c|}{$2(n=5)$} & \\
\hline & 2.10 & \pm & 0.77 & 2.59 & \pm & 1.03 & \\
\hline$P-d(p p m)$ & 54.65 & \pm & 33.93 & 124.17 & \pm & 55.87 & ** \\
\hline $\mathrm{pH}$ & 5.68 & \pm & 0.29 & 6.24 & \pm & 0.43 & * \\
\hline $\mathrm{Al}(\mathrm{ppm})$ & 24.58 & \pm & 7.18 & 22.16 & \pm & 16.40 & Ns \\
\hline $\mathrm{Ca}(\mathrm{ppm})$ & 647.93 & \pm & 286.98 & 787.96 & \pm & 263.59 & Ns \\
\hline $\mathrm{Mg}(\mathrm{ppm})$ & 82.64 & \pm & 17.31 & 132.81 & \pm & 106.77 & Ns \\
\hline K (ppm) & 155.09 & \pm & 22.54 & 155.55 & \pm & 70.06 & Ns \\
\hline $\mathrm{Na}(\mathrm{ppm})$ & 11.50 & \pm & 18.54 & 17.74 & \pm & 9.69 & ** \\
\hline $\mathrm{Cu}$ (ppm) & 2.42 & \pm & 0.64 & 2.13 & \pm & 1.05 & Ns \\
\hline Fe (ppm) & 313.17 & \pm & 113.28 & 580.27 & \pm & 210.03 & $* *$ \\
\hline Mn (ppm) & 80.41 & \pm & 57.04 & 100.89 & \pm & 65.11 & Ns \\
\hline $\mathrm{Zn}(\mathrm{ppm})$ & 5.23 & \pm & 3.05 & 17.49 & \pm & 25.64 & $* *$ \\
\hline $\mathrm{B}(\mathrm{ppm})$ & 0.37 & \pm & 0.17 & 0.36 & \pm & 0.30 & Ns \\
\hline $\mathrm{S}(\mathrm{ppm})$ & - & & & 19.26 & \pm & 8.59 & - \\
\hline
\end{tabular}

MO: Materia orgánica; P-d: Fósforo disponible; -: Valor no determinado; Significancia estadística para el efecto del ciclo: $\mathrm{Ns}=\mathrm{P}>0.05 ; * \mathrm{P}<0.05 ; * * \mathrm{P}<0.01$ 
Tabla 2. Correlaciones Tau de Kendall entre los macro y micronutrientes del sedimento piscícola en tres estanques de cultivo de tilapia roja durante dos ciclos productivos.

\begin{tabular}{|c|c|c|c|c|c|c|c|c|c|c|c|c|c|}
\hline & MO & P-d & $\mathrm{pH}$ & Al & $\mathrm{Ca}$ & Mg & K & $\mathrm{Na}$ & $\mathrm{Cu}$ & $\mathrm{Fe}$ & $\mathrm{Mn}$ & Zn & B \\
\hline P-d (ppm) & $0.31^{*}$ & & & & & & & & & & & & \\
\hline $\mathrm{pH}$ (ppm) & 0.04 & $0.33^{*}$ & & & & & & & & & & & \\
\hline $\mathrm{Al}(\mathrm{ppm})$ & $0.29^{*}$ & -0.14 & $0.37^{*}$ & & & & & & & & & & \\
\hline $\mathrm{Ca}(\mathrm{ppm})$ & $0.49^{* *}$ & $0.36^{* *}$ & $0.31^{*}$ & 0.12 & & & & & & & & & \\
\hline Mg (ppm) & 0.14 & 0.06 & 0.06 & 0.22 & -0.1 & & & & & & & & \\
\hline $\mathrm{K}(\mathrm{ppm})$ & 0.23 & -0.2 & $-0.29^{*}$ & $0.36^{*}$ & 0.06 & 0.2 & & & & & & & \\
\hline $\mathrm{Na}(\mathrm{ppm})$ & $0.29^{\star}$ & 0.18 & 0.1 & 0.08 & $0.35^{*}$ & 0.19 & 0.27 & & & & & & \\
\hline $\mathrm{Cu}(\mathrm{ppm})$ & -0.08 & -0.15 & -0.17 & -6.19 & -0.2 & -0.22 & -0 & $-0.31^{*}$ & & & & & \\
\hline $\mathrm{Fe}(\mathrm{ppm})$ & $0.58^{\star *}$ & $0.37^{* *}$ & 0.21 & 0.01 & $0.32^{*}$ & $0.27^{*}$ & 0.15 & $0.34^{*}$ & -0.02 & & & & \\
\hline Mn (ppm) & -0.04 & -0.1 & -0.08 & 0.06 & -0.1 & 0.15 & $0.32^{*}$ & 0.03 & $0.37^{*}$ & 0.14 & & & \\
\hline Zn (ppm) & $0.47^{* *}$ & $0.46^{* *}$ & $0.28^{*}$ & -0.1 & $0.32^{*}$ & 0.23 & 0.03 & $0.32^{*}$ & 0.01 & $0.71^{* *}$ & 0.15 & & \\
\hline B (ppm) & 0.06 & -0.11 & -0.24 & $0.29^{*}$ & -0.1 & -0.1 & 0.25 & 0.06 & 0.01 & 0.05 & 0.2 & 0.05 & \\
\hline S (ppm) & 0.36 & 0.35 & 0 & 0.08 & 0.28 & -0.19 & 0.03 & 0.15 & 0.28 & 0.22 & 0.11 & $0.49^{*}$ & 0.17 \\
\hline
\end{tabular}

$\mathrm{MO}=$ materia orgánica; $\mathrm{P}-\mathrm{d}=$ fosforo disponible; * significancia al $5 \% ; * *$ significancia al $1 \%$.

hacia el medio ambiente (FAO, 2006), sin embargo, con los resultados de este estudio se corroboró que no hubo diferencia significativa en la concentración de MO entre los dos ciclos productivos (Tabla 1). A pesar de que el tiempo entre los ciclos estudiados fue mayor a un año, y durante este tiempo se continuo cultivando (tres cosechas), no hubo remoción de sedimento y el aporte de nutrientes se mantuvo debido al manejo de aguas verdes en la granja, la concentración de $\mathrm{MO}$ no superó el 3\% en cada ciclo analizado pero hubo un incremento de $23 \%$ entre ciclos. La concentración de MO estuvo dentro del rango óptimo para estanques fertilizados y aceptable para estanques con suministro de alimento comercial de acuerdo a la clasificación establecida por Boyd (2008), y aunque en esta granja se dieron los dos procedimientos simultáneamente, suministro de alimento y fertilización, la baja concentración de MO posiblemente se debió a la alta temperatura del trópico, aunado al uso de probióticos y aireación mecánica, que contribuyen a la degradación de la materia orgánica fresca, resaltando que la descomposición involucra múltiples organismos a distintas escalas espaciales y temporales (Álvarez, 2005) que crean zonas de mayor acumulación. La MO presentó correlación (Tabla 2) positiva y significativa $(\mathrm{P}<0.05)$ con elementos que son producto del manejo en el estanque $(\mathrm{Ca}$, $\mathrm{P}$ y $\mathrm{Na}$ ) y de la composición del suelo en la región ( $\mathrm{Fe}$, Zn y Al), denotando así la característica del sedimento.

El pH ácido durante los dos ciclos (Tabla 1), además de ser característico de los suelos de la región, puede también reflejar los contenidos de $\mathrm{Al}$, Fe y $\mathrm{Mn}$ (que acidifican el suelo), y la descomposición de la $\mathrm{MO}$ que remueve grandes cantidades de oxígeno de la interfase agua-sedimento; adicionalmente, la fertilización y el suministro de urea usado en la granja, también contribuyen con la acidificación del medio. El pH del sedimento se expresa en la columna de agua y en sistemas lenticos, la variación nictimeral del pH está en función de la actividad fotosintética y respiratoria de las comunidades acuáticas (Diemer et al., 2010), esta variación fue representativa en la granja por su sistema de aguas verdes (Yossa et al., 2011).

En el $\mathrm{P}_{-\mathrm{d}}$ entre ciclos hubo diferencia significativa $\mathrm{P}<0.05$ (Tabla 1), posiblemente porque el $\mathrm{P}$ es altamente influenciado por las bacterias, se encuentra en diferentes formas y más del $90 \%$ está en la materia orgánica (Boyd, 1995), el incremento del $P_{-d}$ estuvo correlacionado significativamente con el de la $\mathrm{MO}$ $(\mathrm{P}<0.05)$ (Tablas 1 y 2). Para que el fósforo pueda ser utilizado por el fitoplancton primero debe ser mineralizado por bacterias, pero además requiere temperaturas entre 25 y $30{ }^{\circ} \mathrm{C}$, $\mathrm{pH}$ neutro, humedad y una adecuada relación N:P (Fernández et al., 2006). En esta granja, ubicada en el trópico, donde hay aireación mecánica, suministro de abono, alimento, probióticos, urea y melaza -como fuente de energía para el mantenimiento de fitoplancton-, la dinámica del fósforo es mayor denotándose alta variabilidad (Tabla 1). El pH también contribuye a la dinámica del fósforo porque a $\mathrm{pH}$ ácido aumenta la adsorción del $\mathrm{P}_{-\mathrm{d}}$, lo que sugiere 
que pudo haber mayor absorción durante el primer ciclo, además el $\mathrm{P}$ inorgánico también aporta fósforo soluble que es aprovechado directamente por el fitoplancton, en cuanto que la fracción insoluble constituye la gran reserva de $\mathrm{P}$ inorgánico en el sedimento y reacciona con diversos elementos como $\mathrm{Ca}$, en medio alcalino, $\mathrm{Fe}, \mathrm{Mg}$, e $\mathrm{Al}(\mathrm{OH})$ que precipita ortofosfato, casi inmediatamente (Matijević et al., 2008) en medio ácido, esto se refleja en la correlación del P-d con el pH $(\mathrm{P}<0.05)$ y con $\mathrm{Ca}$, Fe y $\mathrm{Zn}(\mathrm{P}<0.01)$ (Tabla 2$)$.

En los macronutrientes evaluados $\mathrm{Al}, \mathrm{Ca}, \mathrm{Mg}$, $\mathrm{K}$ y $\mathrm{Na}$, no hubo diferencia significativa entre ciclos, con excepción para el $\mathrm{Na}(\mathrm{P}<0.01)$, (Tabla 1); que podría ser resultado de la adición ocasional de sal (cloruro de Potasio) a los estanques para manejo preventivo de enfermedades. Al, $\mathrm{Ca}, \mathrm{K}$ y Mg representan los cationes intercambiables de los cuales Ca representó el mayor valor para los ciclos 1 y 2 respectivamente (Tabla 1 ), tal vez como resultado del encalamiento; además presentó una correlación positiva altamente significativa $\mathrm{P}<0.01$ con la $\mathrm{MO}$ y el $\mathrm{P}_{-\mathrm{d}}$ (Tabla 2), denotando que posiblemente el fósforo es precipitado como fosfato de Calcio. El Al tuvo correlación positiva con $\mathrm{MO}$ y negativa con el $\mathrm{pH}$ pero en ambos casos significativa $(\mathrm{P}<0.05)$ (Tabla 2). En el trópico la acidificación del suelo se debe a la meteorización, que conlleva a bajos contenidos de bases y altas concentraciones de Al y en ocasiones de Fe y Mn (Zapata, 2006); sin embargo estudios realizados por Quirós-Conejo y González-Aguilar (1979) indicaron que al aplicar cal hay una caída brusca en la concentración de $\mathrm{Al}$, contrariamente a lo sucedido en este estudio, donde a pesar de la aplicación de cal, no hubo correlación entre el Ca y Al ( $P>0.05)$ (Tabla 2).

La concentración de $\mathrm{K}$ fue estable durante los dos ciclos productivos (Tabla 1), posiblemente por la adición de cloruro de $\mathrm{K}$ a los estanques; presentó correlación positiva con $\mathrm{Al}$ y negativa con $\mathrm{pH}$ pero en ambos casos significativa $(\mathrm{P}<0.05)$ (Tabla 2). El K además de ser un ión intercambiable con Ca y con $\mathrm{Mg}$, es el elemento absorbido en mayor cantidad, después del $\mathrm{N}$ y el $\mathrm{Ca}$, por las plantas fundamentalmente para el mantenimiento de las aguas verdes (Roldán et al., 2004), sistema de manejo presente en esta granja.

En cuanto a los micronutrientes evaluados, en $\mathrm{Cu}$, $M n$ y $B$ no hubo diferencia significativa $(P<0.05)$ y la concentración de $\mathrm{Cu}$ fue inversa a la de $\mathrm{Mn}$ que se incremento para el segundo ciclo, ya en Fe y Zn la diferencia fue altamente significativa $(P<0.01)$ con incrementos para el segundo ciclo (Tabla 1).

El Fe fue el de mayor concentración en el sedimento y presentó correlaciones positivas altamente significativas $(\mathrm{P}<0.01)$ con $\mathrm{Zn}, \mathrm{MO}$ y $\mathrm{P}_{-\mathrm{d}}$ y significativas $(\mathrm{P}<0.05)$ con $\mathrm{Ca}, \mathrm{Mg}$ y Na (Tabla 2). El Fe es un elemento que se encuentra en altas concentraciones en la corteza terrestre, posiblemente al desprenderse del suelo forma parte de la materia orgánica, y allí sufre una serie de reacciones químicas con otros elementos para formar sustancias más complejas como: fosfato de hierro, carbonato de hierro, óxidos e hidróxidos (Zapata, 2006). Aunque el Fe, es un nutriente esencial para la vida, su concentración no debe exceder de $1.0 \mathrm{mg} / \mathrm{L}$ como Fe total o $0.35 \mathrm{ppm}$ en forma disuelta a fin de prevenir efectos perjudiciales a la vida acuática (Phippen et al., 2008), en este estudio el promedio de Fe en el sedimento fue de $446.72 \mathrm{ppm}$, sin embargo no hubo reportes de manifestaciones clínicas, tal vez porque prevalecen las reacciones de reducción.

El Zn se correlacionó además del Fe, con $\mathrm{MO}$ y $\mathrm{P}$-d $(\mathrm{P}<0.01)$ y con $\mathrm{pH}, \mathrm{Ca}$ y Na $(\mathrm{P}<0.05)$ (Tabla 2$)$. La concentración de $\mathrm{Zn}$ en el sedimento fue en promedio $11 \mathrm{ppm}$, siendo 10 veces inferior a la reportada como contaminante en sedimentos acuáticos en ambiente natural (Márquez et al., 2008); El S, es un micronutriente proveniente de la meteorización del suelo, además de ser adicionado en forma de yeso $\mathrm{CaSO}_{4}\left(2 \mathrm{H}_{2} \mathrm{O}\right)$ al cultivo, lo que pudo influenciar en el alto valor (Tabla 1) determinado a pesar del uso de probióticos, este hecho contrasta con los valores reportados por Hana et al., (2008) de azufre en cultivos de camarón con y sin probióticos que no superaron el $5 \%$.

Con el valor nutricional del sedimento entre ciclos productivos se corroboró la dinámica en el reciclaje de nutrientes, ya que en esta granja se suministró constantemente aditivos para mantener el sistema de aguas verdes, sin embargo, la MO, el P-d, y los macro y micro nutrientes del sedimento en términos generales estuvieron estables. Este hecho podría deberse a los subsidios tecnológicos como el uso de aireadores, la actividad biológicamente eficiente de los probióticos y la acción bioturbadora y filtradora de la especie cultivada. Así el sedimento de esta granja no representaría un riesgo ambiental, aunque su uso como abono agrícola tampoco sería aconsejable debido a la baja composición nutricional, principalmente en MO. En aras de contribuir con el equilibrio en el sistema de cultivo, es importante monitorear y verificar el sinergismo o antagonismo entre los aditivos suministrados.

\section{Agradecimientos}

Al Ministerio de Agricultura y Medio Ambiente (MADR), Universidad de los Ilanos y Aquicultura Primavera Ltda., por el financiamiento del proyecto Dinámica de Nutrientes en estanques piscícolas, convenio 057/2007 Unillanos-MADR. 


\section{Referencias}

Adámek Z, Maršálek B. 2012. Bioturbation of sediments by benthic macroinvertebrates and fish and its implication for pond ecosystems: a review. Aquaculture International, 1-17.

Adedeji AA, Adeniyi IF, Adetokunbo OR. The sediment characteristic and benthic macroinvertebrate fauna of some fish ponds in Ife north local government area (LGA), Nigeria. International Journal of Fisheries and Aquaculture, 2012; 4(1): 7-12.

Álvarez S. La descomposición de materia orgánica en humedales: la importancia del componente microbiano. Ecosistemas, 2005; 14(2): 17-29.

APHA. 2005. Standard methods for the examination of water and wastewater, 21st ed. American Public Health Association, Washintong D.C.

Avnimelech Y, Ritvo G. Shrimp and fish pond soils: processes and management. Aquaculture, 2003; 220(1-4): 549-567.

Botero. 1999. Paisajes fisiográficos de Orinoquia-Amazonia(ORAM) Colombia. Ministerio de Hacienda y Crédito Público, Instituto Geográfico Agustín Codazzi, Subdirección de Investigación y Divulgación Geográfica.p 361

Boyd CE. 1995. Soil nutrients. En: Bottom soils sediment, and pond aquaculture (Hall, Ed.). Chapman y Hall, United State of America. p 69-137.

Boyd EC 2008. Pond Bottom Soil Analyses, Global Aquaculture Advocate. Department of Fisheries and Allied Aquacultures, Auburn University, pp. 91-92.

Boyd EC, Woods CW, Thunjai T. 2002. Aquaculture Pond Bottom Soil Quality Management. Pond Dynamics/Aquaculture Collaborative Research Support Program. Oregon State University, Corvallis. pp 41.

Diemer O, Neu DH, Feiden A, Lorenz EK, Bittencourt F, Boscolo WR. Dinâmica nictimeral e vertical das características limnológicas em ambiente de criação de peixes em tanques-rede $\mathrm{Ci}$. Anim. Bras, 2010; 11(1):24-31.

Espinal C, Martínez HFG 2005. La cadena de la piscicultura en Colombia: Una mirada global de su estructura y dinámica 1991-2005, Observatorio Agrocadenas Colombia. MADR, pp. 41.

FAO. 2006. El estado mundial de la pesca y la acuicultura. Organización de las naciones unidas para la agricultura y la alimentación, Roma PAGINAS.

Fernández CL, Mendoza R, Vázquez S. Fracciones de fósforo en suelos de corrientes con producción citrícola, arrocera y pastoril. Cienc. suelo 2006; 24(2):161-168.

Förstner U. 1990. Roof: A source of pollutants in urban drainage systems. Proceedings of Fifth International Conference on Urban Storm Drainage, Suita, Osaka, Japan, pp. 469-474.

Gu Q, Xiong B, Zhu Y, Yang X, Shi P. Comparison of benthic macroinvertebrates in three polyculture models of ponds stocking mainly Ctenopharyngodon idellus. Desalination and Water Treatment, 2012; 45(1-3):26-39.
Ihejirika CE, Onwudike SU, Nwaogu LA, Emereibeole E I, Ebe T E, Ejiogu CC. Assessment of aquaculture sediment for agricultural fertilizer supplement and soil conditioner in Owerri Urban, Nigeria. Journal of Research in Agriculture, 2012; 1(1):34-38.

Jiménez-Tenorio N, Morales-Caselles C, Kalman J, Salamanca MJ, González de Canales ML, Sarasquete C, DelValls T Á. Determining sediment quality for regulatory proposes using fish chronic bioassays. Environment International, 2007; 33(4): 474-480.

Krebs RL 2003. Respiración del suelo como herramienta para evaluar calidad de fondos en acuicultura. I. Desarrollo de un protocolo estándar para medir dióxido de carbono, Facultad de ingeniería marítima y ciencias del mar. Escuela superior politécnica del litoral, Ecuador, pp. 67.

Lehmann M. 2005. Potencial redox em sedimentos de viveiros de água doce e salgada: metodologia de determinação e comportamento Universidade Federal de Santa Catarina, Florianópolis - Santa Catarina, pp. 36.

Leue M, Lang F. Recycling soil nutrients by using channel deposits as fertilizers? Nutrient Cycling in Agroecosystems, 2012; 93(1):75-88.

MADR, 2010. Pesca y Acuicultura Colombia 2009 "Informe técnico regional de cuencas del Orinoco y Amazonas". Corporación Colombiana Internacional, pp. 71.

Márquez A, Senior W, Martínez G, Castañeda J, González Á. Concentraciones de metales en sedimentos y tejidos musculares de algunos peces de la laguna de Castillero, Venezuela,. Revista Científica, FCV-LUZ XVIII 2008; (2):121-133.

Matijević S, Kušpilić G, Kljaković-Gašpić Z, Bogner D. Impact of fish farming on the distribution of phosphorus in sediments in the middle Adriatic area. Marine pollution bulletin, 2008; 56: 535-548.

Meseck S, Cutter G. Selenium Behavior in San Francisco Bay Sediments. Estuaries and Coasts, 2012; 35(2): 646-657.

Parra JP, Espinosa LF. Distribución de metales pesados $(\mathrm{Pb}, \mathrm{Cd}$ y $\mathrm{Zn}$ ) en perfiles de sedimento asociado a rhizophora mangle en el río Sevilla - Ciénaga Grande de Santa Marta, Colombia. Bol. Invest. Mar. Cost, 2008; 37(1): 95-110.

Phippen B, Horvath C, Nordin R, Nagpal N. 2008. Ambient water quality guidelines for iron: overview. Ministry of environment province of british columbia, British Columbia.

Poleo G, Aranbarrio JV, Mendoza L, Romero O. Cultivo de cachama blanca en altas densidades y en dos sistemas cerrados. Pesquisa Agropecuaria Brasileira, Brasilia, 2011; 46(4): 429-437.

Quin GP, Keough MJ. 2002. Experimental designs and data analysis for biologist. Cambridge University Press.pp. 537.

Quirós-Conejo S, González-Aguilar MA. Neutralización del aluminio intercambiable y aprovechamiento del fósforo en tres suelos de Costa Rica. Organización para Estudios Tropicales, 1979; 3(2): 137-149.

Roldán MF, Venialgo CA, Gutiérrez NC. 2004. Potasio disponible, de reserva y energía de reemplazamiento en suelos y el nivel foliar en rye-grass. Universidad Nacional del Nordeste (Argentina). Comunicaciones Científica y Tecnológicas, A-072(1): 1-3. 
Steeby JA, Hargreaves JA, Tucker CS, Kingsbury S. Accumulation, organic carbon and dry matter concentration of sediment in commercial channel catfish ponds. Aquacultural Engineering, 2004; 30(1):115-126.

Vita R, Marín A, Madrid JA, Jiménez-Brinquis B, César A, Marín-Guirao L. Impacto ambiental de la acuicultura en el bentos marino: experimentos de exclusión - inclusión. Boletin Institucional Español de Oceanografía, 2002; 18(1-4): 75-86.
Yossa MI, Hernández G, Vásquez-Torres W. 2011. Correlación entre la dinámica de nutrientes y calidad del agua en estanques piscícolas comerciales de tilapia roja (Oreochromis sp) y cachama blanca (Piaractus brachypomus). Informe técnico convenio 057/2007. Universidad de los Llanos/ Ministerio de Agricultura y Desarrollo Rural, p. 71.

Zapata HR. 2006. Química de los procesos podogenéticos, Universidad Nacional de Medellin, pp. $63-65$. 